\title{
La gestione del paziente con lipodistrofia
}

\author{
Giovanni Ceccarini ${ }^{1} \cdot$ Silvia Migliaccio $^{2} \cdot$ Ferruccio Santini $^{1}$
}

Accettato: 13 giugno 2020 / Pubblicato online: 1 giugno 2021

(C) The Author(s) 2021

\section{Introduzione}

Le sindromi lipodistrofiche costituiscono un gruppo eterogeneo di malattie caratterizzate dalla perdita più $o$ meno estesa di tessuto adiposo sottocutaneo, in assenza di uno stato di deprivazione nutrizionale o di aumentato catabolismo.

Esistono oltre quaranta forme diverse di lipodistrofia e per una dettagliata analisi dei vari sottotipi rimandiamo alle più recenti rassegne e linee guida sul tema [1-5].

In base all'eziologia, le lipodistrofie vengono distinte in genetiche $o$ acquisite e, in base al grado di perdita del tessuto adiposo, in generalizzate o parziali (Fig. 1) [3]. Le forme genetiche includono sottotipi a trasmissione autosomico recessiva e dominante, mentre le forme acquisite riconoscono di solito una causa autoimmune con l'eccezione di quelle causate dalla terapia antiretrovirale, in pazienti HIV positivi o quelle (estremamente) localizzate causate dall'iniezione sottocutanea di insulina o di altri farmaci [3].

La prevalenza di lipodistrofia (escludendo la lipodistrofia associata a HIV) è stimata attorno a 3,07 casi per milione di individui $(0,23$ casi per milione per quanto riguarda le forme generalizzate e 2,84 casi per quanto riguarda le forme parziali) [3].

Nei soggetti lipodistrofici, l'espandibilità del tessuto adiposo e la sua capacità di accumulare un surplus energetico vengono superate anche per apporti calorici minimi. Questo comporta da un lato un accumulo di lipidi in siti ectopici quali fegato, muscolo, rene e pancreas e, dall'altro, la croni-

\section{$凶$ G. Ceccarini}

giovanni.ceccarini@unipi.it

1 Centro Obesità e Lipodistrofie, U.O. di Endocrinologia I, Azienda Ospedaliero Universitaria di Pisa, Pisa, Italia

2 Dipartimento di Scienze del Movimento, Umane e della Salute, Università Foro Italico di Roma, Roma, Italia ca riduzione dei livelli circolanti dell'ormone leptina. Bassi livelli di leptina stimolano il senso di fame, alimentando un circolo vizioso che porta a un progressivo accumulo ectopico di grasso. A livello periferico viene meno l'azione che favorisce l'utilizzo del glucosio e degli acidi grassi da parte del muscolo scheletrico con conseguente lipotossicità. L'accumulo ectopico di grasso intramuscolare ed epatico contribuisce allo sviluppo di resistenza insulinica.

Le sindromi lipodistrofiche sono quindi caratterizzate da molteplici alterazioni ormonali e metaboliche, quali insulino-resistenza con precoce comparsa di diabete mellito, ipertrigliceridemia grave, steatosi epatica non alcolica (NAFLD) e un quadro analogo a quello dell'ovaio policistico [1]. In alcuni pazienti tali complicanze possono condurre a nefropatia e retinopatia diabetica, pancreatite acuta dovuta alla grave ipertrigliceridemia e chilomicronemia, cirrosi epatica e patologie cardiovascolari a insorgenza precoce. In alcuni sottotipi di lipodistrofia sono più frequenti cardiomiopatie e nefropatie, anomalie della conduzione cardiaca, miopatie, alterazioni ossee e un aumentato rischio di neoplasia.

Inoltre, il paziente lipodistrofico lamenta spesso sintomi somatici con dolore predominante e riduzione del tono dell'umore, fenomeni non ancora ben caratterizzati dal punto di vista fisiopatologico.

\section{Diagnosi delle lipodistrofie}

La presenza di lipodistrofia può essere sospettata in soggetti con assenza parziale o generalizzata di tessuto adiposo e convalidata da misure antropometriche come la plicometria e/o esami strumentali come la densitometria a doppio raggio fotonico (DXA) e la risonanza magnetica total-body (MRI). La presenza di specifiche caratteristiche fisiche, anamnestiche e la presenza di comorbidità possono incrementare il so- 


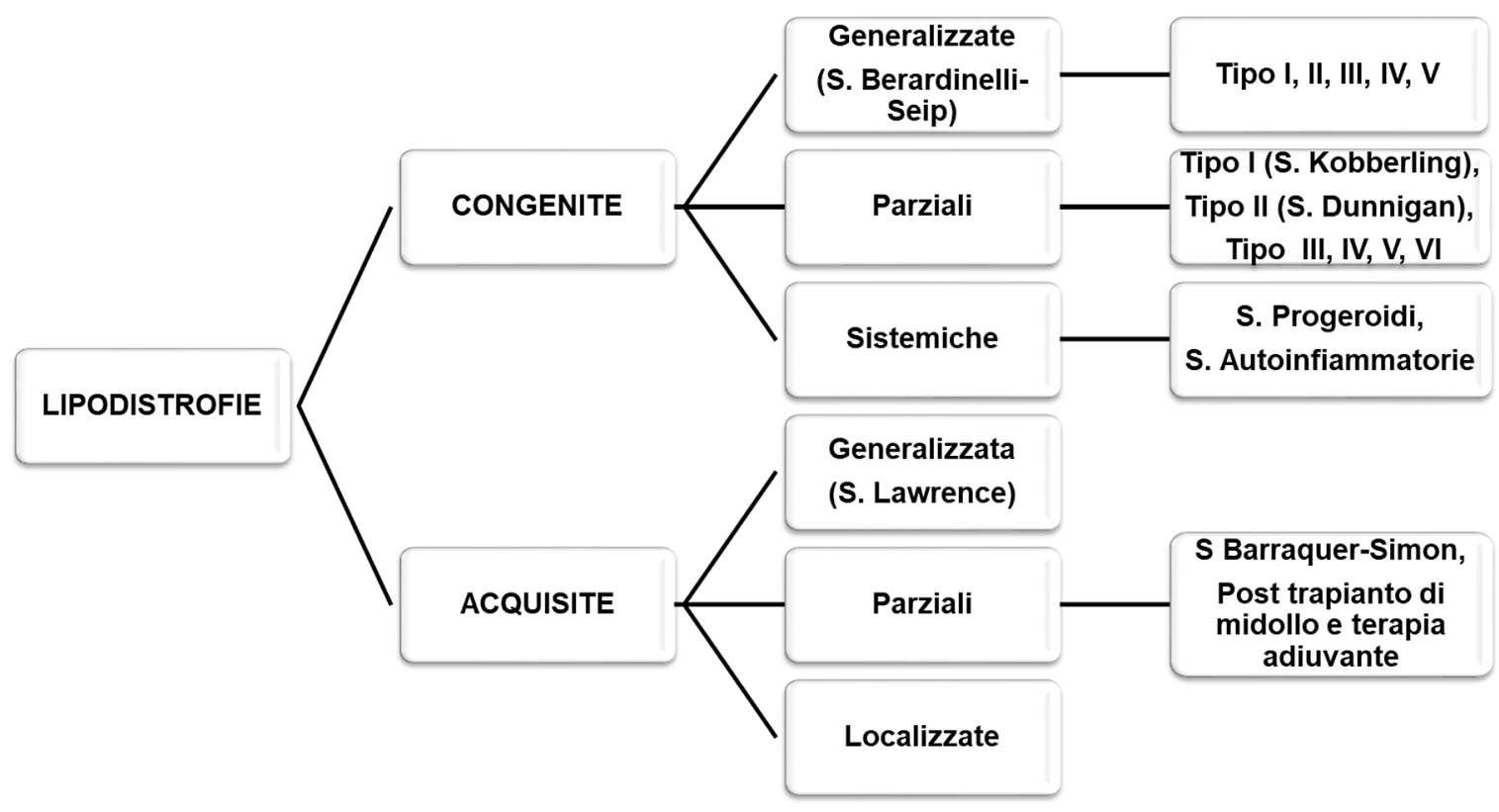

Fig. 1 Classificazione delle principali forme di lipodistrofia

Tabella 1 Caratteristiche che fanno sospettare un caso di lipodistrofia e condizioni che entrano in diagnosi differenzia con la malattia

Caratteristica essenziale

Perdita di tessuto adiposo generalizzata o parziale

Caratteristiche cliniche di supporto alla diagnosi

- Marcata insulino-resistenza o diabete mellito di tipo 2

- Acanthosis nigricans, sindrome dell'ovaio policistico

- Ipertrigliceridemia

- Storia di pancreatiti associate a ipertrigliceridemia

- Epatomegalia e/o steatosi epatica e/o steatoepatite

- Pseudoipertrofia muscolare

- Flebomegalia

- Iperfagia, fame difficilmente controllabile

- Aspetto pseudo-acromegaloide o progeroide

- Accumulo di tessuto adiposo a livello del collo

Diagnosi differenziale

- Diabete mellito scompensato

- Anoressia nervosa, cachessia e malnutrizione

- Infezioni croniche

- Insufficienza surrenalica

- Tireotossicosi

- Sindrome diencefalica

- Sindrome di Cushing

- Obesità centrale

- Lipodistrofie associate a terapia anti-retrovirale

- Acromegalia

spetto di lipodistrofia (Tab. 1) [4]. I livelli di leptina non sono determinanti per la diagnosi. Nel caso di sospetto clinico ben definito possono essere richiesti i relativi test genetici: la genotipizzazione può includere un sequenziamento limitato a un gene o a un gruppo di geni candidati. La diagnosi differenziale include condizioni che si presentano con grave calo ponderale (malnutrizione, anoressia nervosa, diabete mellito non controllato, insufficienza corticosurrenalica, cachessia neoplastica, atrofia associata a HIV, infezioni croniche) [5]. Le lipodistrofie generalizzate possono essere confuse anche con l'acromegalia, mentre le lipodistrofie parziali possono entrare in diagnosi differenziale con la sindrome di Cushing o con forme di obesità centrale (Tab. 1).

\section{Terapia}

Il trattamento delle lipodistrofie ha lo scopo di migliorare innanzitutto le complicanze metaboliche associate alla patologia, dato che mortalità e morbidità risultano attenuate da un intervento precoce. La dieta ipocalorica a basso contenuto lipidico e l'esercizio fisico sono parte integrante del piano terapeutico [2]. La dieta può essere difficile da seguire perché i pazienti, in particolare nelle forme generalizzate, sono in genere iperfagici a causa della carenza di leptina. L'esercizio fisico può migliorare la sensibilità insulinica ma è controindicato in pazienti con grave cardiomiopatia.

La metformina è il farmaco di prima scelta nel trattamento del diabete e dell'insulino-resistenza [2]. I tiazolidinedio- 
ni sono stati impiegati nelle forme parziali di lipodistrofia ma il loro utilizzo è dibattuto [2]. Nei casi in cui non si raggiunga un adeguato controllo glicemico, è indicato passare alla terapia insulinica. Per l'ipertrigliceridemia è necessario aumentare l'apporto di acidi grassi polinsaturi (omega 3) da associare eventualmente a fibrati [2].

La Metreleptina (leptina umana ricombinante) migliora le alterazioni metaboliche dei pazienti con lipodistrofia generalizzata: riduce l'iperfagia con conseguente calo ponderale, riduce la glicemia a digiuno e determina una riduzione dei livelli di emoglobina glicata (Hb A1c) mediamente del 2\% dopo un anno di trattamento [2]. La Metreleptina riduce, inoltre, i livelli di trigliceridi fino al 60\% e migliora la steatosi epatica riducendo il contenuto epatico di lipidi e attenuando l'infiammazione [2]. Favorisce inoltre la regolarizzazione del ciclo mestruale e può abbassare la proteinuria, riducendo l'iperfiltrazione glomerulare tipica di alcune forme [2].

Il trattamento delle complicanze associate a lipodistrofia mediante Metreleptina è approvato dall'Agenzia Europea del Farmaco (EMA). In Italia, dal maggio 2020, il trattamento viene dispensato dal sistema sanitario nazionale, esclusivamente per le forme generalizzate, a seguito di piano terapeutico ospedaliero.

Infine, i cambiamenti estetici causati dalla lipodistrofia possono portare a disagio psicologico. La chirurgia plastica può contribuire a migliorare l'aspetto estetico e i possibili interventi includono il trapianto autologo di tessuto adiposo, fillers dermici o innesti per trattare la lipoatrofia facciale, la liposuzione delle aree con eccessivo deposito adiposo (come il collo) e l'impianto di protesi mammarie nei casi di ipoplasia.

\section{Conclusioni}

La lipodistrofia è una malattia rara, poco conosciuta e sottodiagnosticata. La presenza di centri di riferimento è necessaria per un corretto inquadramento e la gestione ottimale di questi pazienti. Lo studio di queste malattie rare ha subito recentemente un grande impulso e la costituzione di un registro europeo [6], a cui afferiscono i principali Centri internazionali, contribuirà ad accelerare l'acquisizione delle conoscenze. La costituzione da parte della Società Italiana di Endocrinologia (http://www.societaitalianadiendocrinologia.
it/html/cnt/Lipodistrofie.asp) di un gruppo di studio sulle lipodistrofie è in linea con questa necessità.

Funding Note Open access funding provided by Università di Pisa within the CRUI-CARE Agreement.

Conflitto di interesse Gli autori Giovanni Ceccarini, Silvia Migliaccio e Ferruccio Santini dichiarano di aver ricevuto compensi per consulenze da parte dell'Azienda Farmaceutica Amryt che commercializza la leptina umana ricombinante.

Consenso informato Lo studio presentato in questo articolo non ha richiesto sperimentazione umana.

Studi sugli animali Gli autori di questo articolo non hanno eseguito studi sugli animali.

Nota della casa editrice Springer Nature rimane neutrale in riguardo alle rivendicazioni giurisdizionali nelle mappe pubblicate e nelle affiliazioni istituzionali.

Open Access This article is licensed under a Creative Commons Attribution 4.0 International License, which permits use, sharing, adaptation, distribution and reproduction in any medium or format, as long as you give appropriate credit to the original author(s) and the source, provide a link to the Creative Commons licence, and indicate if changes were made. The images or other third party material in this article are included in the article's Creative Commons licence, unless indicated otherwise in a credit line to the material. If material is not included in the article's Creative Commons licence and your intended use is not permitted by statutory regulation or exceeds the permitted use, you will need to obtain permission directly from the copyright holder. To view a copy of this licence, visit http://creativecommons.org/licenses/by/4.0/.

\section{Bibliografia}

1. Garg A (2011) Clinical review: lipodystrophies: genetic and acquired body fat disorders. J Clin Endocrinol Metab 96(11):3313-3325

2. Brown RJ, Araujo-Vilar D, Cheung PT et al (2016) The diagnosis and management of lipodystrophy syndromes: a multi-society practice guideline. J Clin Endocrinol Metab 101(12):4500-4511

3. Araujo-Vilar D, Santini F (2019) Diagnosis and treatment of lipodystrophy: a step-by-step approach. J Endocrinol Invest 42(1):61-73

4. Handelsman Y, Oral EA, Bloomgarden ZT et al (2013) The clinical approach to the detection oflipodystrophy - an AACE consensus statement. Endocr Pract 19(1):107-116

5. Foss-Freitas MC, Akinci B, Luo Y et al (2020) Diagnostic strategies and clinical management of lipodystrophy. Expert Rev Endocrinol Metab 15(2):95-114

6. von Schnurbein J, Adams C, Akinci B, Ceccarini G et al (2020) European lipodystrophy registry: background and structure. Orphanet J Rare Dis 15(1):17 